\title{
Teaching Virtual Work without the Abstract Concepts
}

\section{Dr. Barry T. Rosson P.E., Florida Atlantic University}

Dr. Barry Rosson is a Professor of Structural Engineering at Florida Atlantic University. Prior to joining FAU, he was a professor at the University of Nebraska for 16 years. His areas of research interest are in nonlinear structural mechanics, numerical methods, structural dynamics and steel structures. He has received numerous campus and college-wide teaching and service awards. At the national level of the American Society of Civil Engineers, he has served as the Chair of the society-wide Committee on Professional Practice, President of the Architectural Engineering Institute, and Associate Editor of the Journal of Structural Engineering. He is a Fellow of both ASCE and AEI and is a Registered Professional Engineer. Prior to working as a professor, he was a consulting engineer at Ellisor and Tanner, Inc. in Dallas, Texas. 


\title{
Teaching Virtual Work Without the Abstract Concepts
}

\author{
Barry T. Rosson
}

\begin{abstract}
This paper presents a straight-forward approach for teaching the subject of virtual work that is consistent with other presentations in mechanics in which the total external work and total strain energy develop as a result of real loads being applied to a structure. When the virtual work method is introduced to students in this way, they are given the opportunity to understand why the method works without having to consider the typical abstract concepts that are usually associated with the subject. By considering two loading sequences of external loads with increasing complexity, a planar truss is used to illustrate how the generalized virtual work expression can be developed and explained using real external work and strain energy principles. Survey results of students' understanding of why the method works using the traditional virtual work approach versus the new approach are presented and discussed.
\end{abstract}

\section{Introduction}

The principle of virtual work has been known since it was first introduced by John Bernoulli in 1717 , and ever since it has been widely used in a variety of applications in mechanics. It is generally considered to be an advanced topic, and although most students have usually had one or more Physics courses where work and energy principles were routinely discussed, it is seldom taught in Mechanics of Materials courses. Considering the subject to be too advanced and difficult to understand, instruction of the subject is often delayed for inclusion in Structural Analysis courses. In most textbooks, the subject is introduced using an imaginary or virtual force to produce internal virtual loads that ride along the real displacements ${ }^{1-9}$. Whereas there is nothing technically wrong with this description, students are instantly thrown off by the foreign terminology that is inconsistent with the presentations on other subjects in mechanics. With a way of teaching virtual work as a straightforward and logical extension of real external work and strain energy principles, students find the material to be easier to understand and learn why the method works, instead of just how to use it.

The focus of this paper is to provide a way of teaching the principle of virtual work that does not rely on using a virtual external unit load from the start. Instead, the principle is presented using real loads that are applied to a real structure using two separate force-time functions and two separate load sequences. The principle is explained by carefully presenting the total external work and total strain energy equations beginning first with a single load $\mathrm{P}$ applied to a planar truss with one load sequence. Then loads $\mathrm{P}$ and $\mathrm{Q}$ are applied using two load sequences in which the load Q is applied at the location and in the direction of the desired displacement. From this basis of understanding, an additional load $\mathrm{S}$ is included in both load sequences to discuss its influence on the displacement expression. This leads to a general understanding of the influence that any number of additional loads would have on the displacement expression, and that the effect of the load Q remains unchanged as these loads are applied. It then becomes evident that 
the desired displacement due to all the applied loads except Q can be determined directly from the derived expression, and as a result, Q does not actually need to be one of the loads from the actual loading condition of the structure - it can exist solely as a convenience to find the desired displacement. Since the load Q has this property, there is no requirement for it to actually be a "unit load", and it can be placed anywhere on the structure and at any orientation where a displacement is desired.

\section{Displacement at Joint 4 Due to Load P}

For the truss with linear-elastic material properties in Figure 1, and with the load applied at joint 4 according to the loading sequence in Figure 2, the external work that is done from $t_{0}$ to $t_{1}$ is given as

$$
W=\frac{1}{2} P \Delta_{44}
$$

where $\Delta_{44}$ is the vertical displacement at joint 4 due to the load $\mathrm{P}$ at joint 4 .

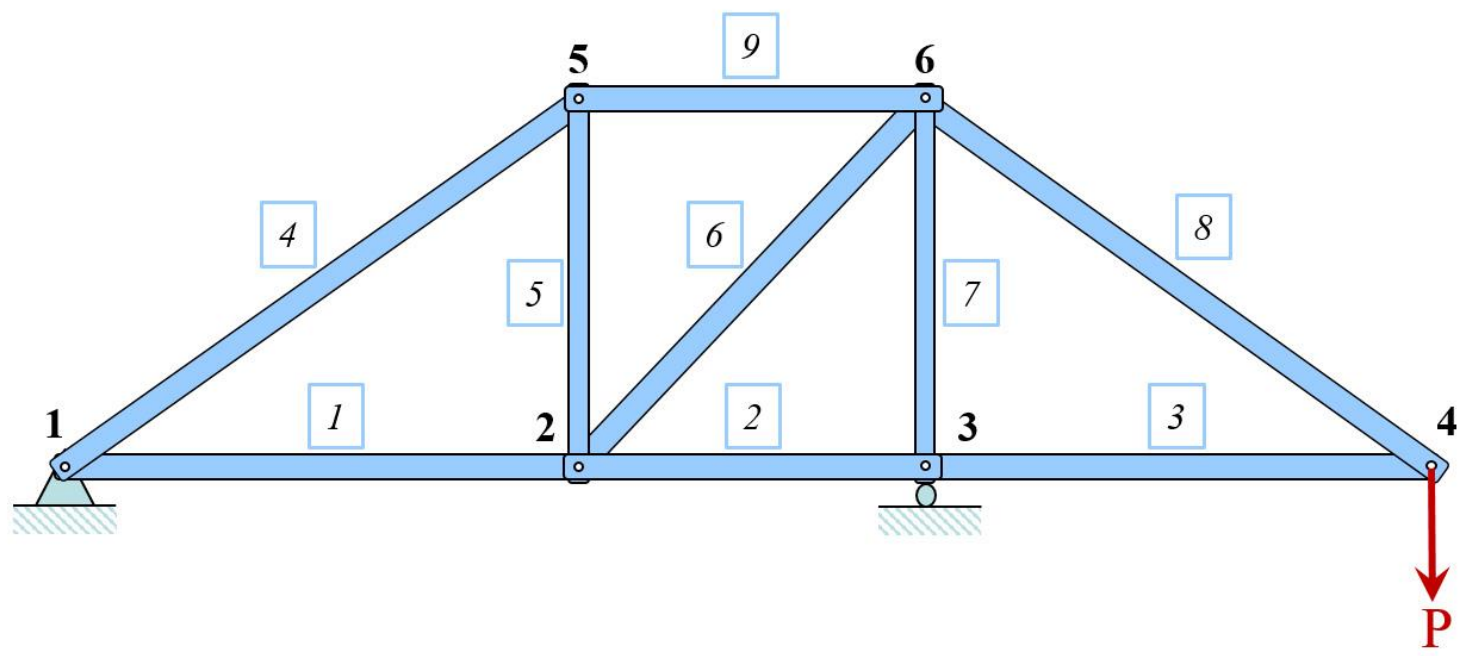

Figure 1. Truss with load P.

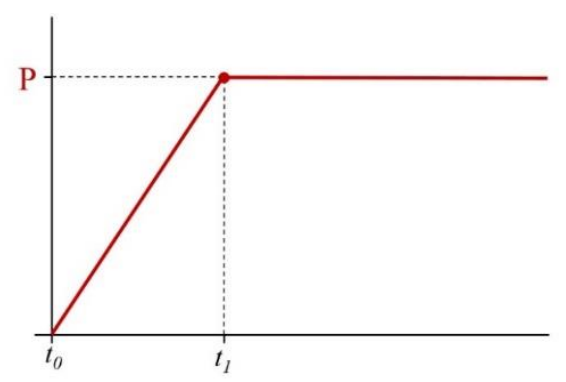

Load Sequence I

Figure 2. Load P applied with Load Sequence I. 
The strain energy stored in the nine members of the truss from $t_{0}$ to $t_{1}$ is given as

$$
U=\frac{1}{2} \sum_{j=1}^{9} F_{j}^{P} \delta_{j}^{P}
$$

where $F_{j}^{P}$ is the internal force in member $j$ due to the load $\mathrm{P}$. The term $\delta_{j}^{P}$ is the change in length of member $j$ due to $F_{j}^{P}$ and is determined using

$$
\delta_{j}^{P}=\frac{F_{j}^{P} L_{j}}{A_{j} E_{j}}
$$

(Appendix A provides a detailed development of Equations (1) through (3) for the truss and loading condition given in Figures 1 and 2.)

Due to conservation of energy $(U=W)$, Equations (1) and (2) can be used to find the vertical displacement at joint 4 due to the load $\mathrm{P}$ at joint $4(\Delta 44)$.

$$
\frac{1}{2} \sum_{j=1}^{9} F_{j}^{P} \delta_{j}^{P}=\frac{1}{2} P \Delta_{44}
$$

This illustrates the use of external work and strain energy to find the displacement at a joint; however, it is limited to the specific case in which there is only one external load, and it must be applied in the same direction and at the location of the desired displacement. In general, we would like to be able to find the displacement in any direction at any joint, and to also have any number of external loads applied to our truss. For the moment we will ignore the multiple external loads, and consider the horizontal displacement at joint 6 in Figure 1 to be the desired displacement. Using only external work and strain energy equations, an expression will be developed to determine the horizontal displacement at joint 6 that is due entirely to the load $\mathrm{P}$ at joint $4\left(\Delta_{64}\right)$.

\section{Displacement at Joint 6 Due to Load P}

By introducing the horizontal load Q in Figure 3, and the load sequences in Figure 4, we can find an expression for the displacement $\Delta_{64}$ that is due only to the load P. In Figure 4 , notice at $t_{2}$ and beyond the loads P and Q are fully applied for both load sequences I and II. Because of this condition at $t_{2}$ and beyond, each member has an axial force and corresponding strain from load sequence I that is equal to the member's axial force and strain from load sequence II. Thus, the stain energy of load sequence I is equal to the strain energy of load sequence II $\left(U_{I}=U_{I I}\right)$ at $t_{2}$ and beyond. Since the strain energy is equal to the total external work for each loading sequence ( $U_{I}=W_{I}$ and $U_{I I}=W_{I I}$ ), we can equate the strain energy from load sequence I with the total external work from load sequence II $\left(U_{I}=W_{I I}\right)$ at $t_{2}$ and beyond. It is this relationship that will allow us to find an expression for the displacement $\Delta_{64}$ that is due entirely to the load P. 


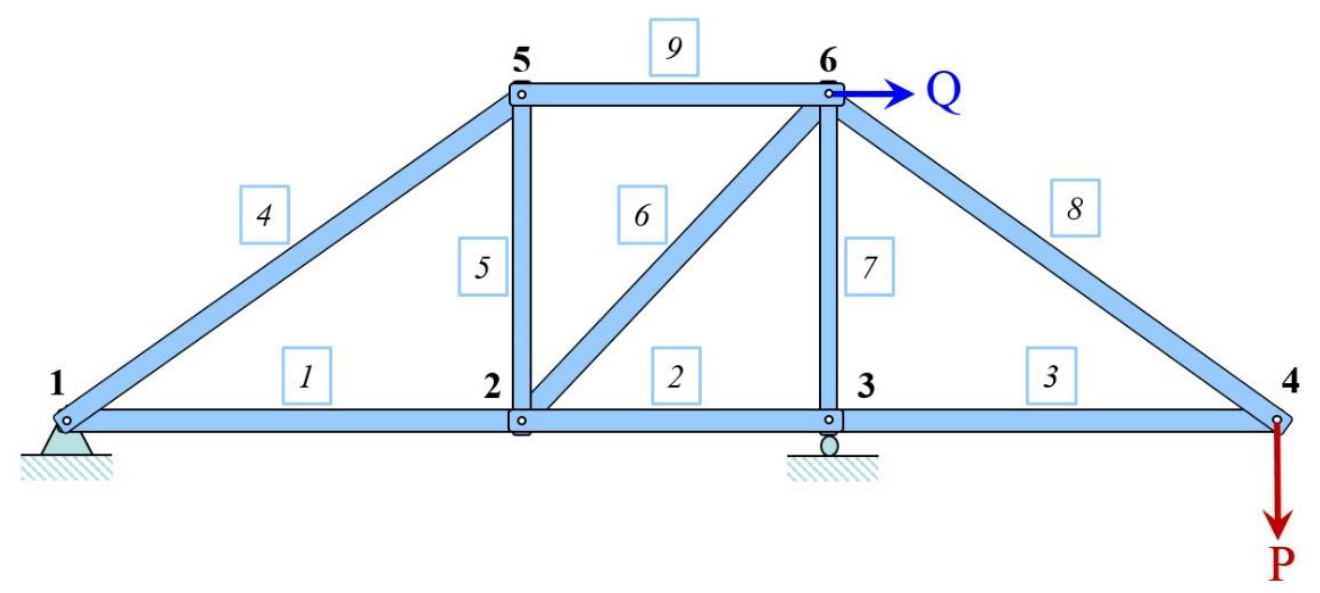

Figure 3. Truss with loads $\mathrm{P}$ and Q.

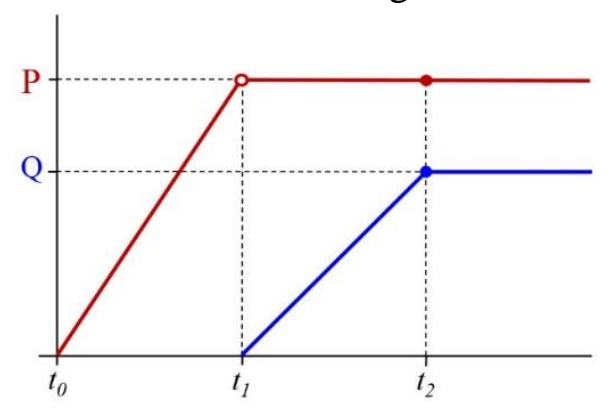

Load Sequence I

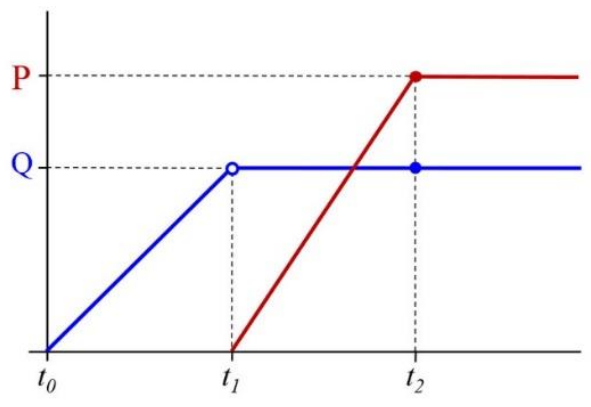

Load Sequence II

Figure 4. Loads P and Q applied with Load Sequences I and II.

First, considering load sequence I, the strain energy stored in all nine members from $t_{0}$ to $t_{2}$ is

$$
U_{I}=\frac{1}{2} \sum_{j=1}^{9} F_{j}^{P} \delta_{j}^{P}+\frac{1}{2} \sum_{j=1}^{9} F_{j}^{Q} \delta_{j}^{Q}+\sum_{j=1}^{9} F_{j}^{P} \delta_{j}^{Q}
$$

where $F_{j}^{Q}$ is the internal force in member $j$ due to the load Q and $\delta_{j}^{Q}$ is the change in length of member $j$ due to $F_{j}^{Q}$. The third term in Equation (5) results from the fact that the load $\mathrm{P}$ is already fully applied from $t_{1}$ to $t_{2}$, and the resulting member forces $F_{j}^{P}$ (due to load $\mathrm{P}$ ) are multiplied by the change in member length values $\delta_{j}^{Q}$ (due to load Q) to account for this portion of the strain energy. (Appendix B provides a detailed development of Equation (5) for the truss and loading conditions given in Figures 3 and 4.)

Next, considering load sequence II, the total external work that is done from $t_{0}$ to $t_{1}$ is

$$
W_{I I}=\frac{1}{2} Q \Delta_{66}
$$


where $\Delta_{66}$ is the horizontal displacement at joint 6 due to the load Q at joint 6 . The total external work that is done from $t_{0}$ to $t_{2}$ is

$$
W_{I I}=\frac{1}{2} Q \Delta_{66}+\frac{1}{2} P \Delta_{44}+Q \Delta_{64}
$$

where $\Delta_{64}$ is the horizontal displacement at joint 6 due to load $\mathrm{P}$ at joint 4 . The third term in Equation (7) results from the fact that the load Q is already fully applied from $t_{1}$ to $t_{2}$, and it is multiplied by the displacement $\Delta_{64}$ (due to load P) to account for this portion of the external work. (Appendix B provides a detailed development of Equation (7) for the truss and loading conditions given in Figures 3 and 4.)

The application of the load Q for load sequence II from $t_{0}$ to $t_{1}$ is very similar to that for load $\mathrm{P}$ in Figures 1 and 2 from $t_{0}$ to $t_{1}$. Thus, just as with Equation (4), conservation of energy provides the following relationship

$$
\frac{1}{2} \sum_{j=1}^{9} F_{j}^{Q} \delta_{j}^{Q}=\frac{1}{2} Q \Delta_{66}
$$

As previously mentioned, we equate the strain energy from load sequence I with the total external work from load sequence II $\left(U_{I}=W_{I I}\right)$ to find an expression for the horizontal displacement $\Delta_{64}$ that is due entirely to the load $\mathrm{P}$.

Using Equation (5) for $U_{I}$, and Equation (7) for $W_{I I}$, we obtain the following relationship

$$
\frac{1}{2} \sum_{j=1}^{9} F_{j}^{P} \delta_{j}^{P}+\frac{1}{2} \sum_{j=1}^{9} F_{j}^{Q} \delta_{j}^{Q}+\sum_{j=1}^{9} F_{j}^{P} \delta_{j}^{Q}=\frac{1}{2} Q \Delta_{66}+\frac{1}{2} P \Delta_{44}+Q \Delta_{64}
$$

Due to the equalities given in Equations (4) and (8), this expression simplifies to the following relationship

$$
\sum_{j=1}^{9} F_{j}^{P} \delta_{j}^{Q}=Q \Delta_{64}
$$

The desired displacement $\Delta_{64}$ can now be determined directly from Equation (10). Although the load Q appears in the equation, the displacement $\Delta_{64}$ is due entirely to the load P. The internal member forces $F_{j}^{P}$ are determined from an analysis of the truss due to the load $\mathrm{P}$, and the change in member lengths $\delta_{j}^{Q}$ are determined from an analysis of the truss in which the internal member forces $F_{j}^{Q}$ are due to the load Q.

$$
\delta_{j}^{Q}=\frac{F_{j}^{Q} L_{j}}{A_{j} E_{j}}
$$




\section{Displacement at Joint 6 Due to Loads $P$ and $S$}

Equation (10) provides a convenient way to find a displacement at a desired location that is due to a single load at a separate location. However, it is common to have multiple external loads on a truss, and thus a more generalized equation is needed for this loading condition. The truss in Figure 5 has the same P and Q loads as in Figure 3, but an additional load S has been added to joint 2.

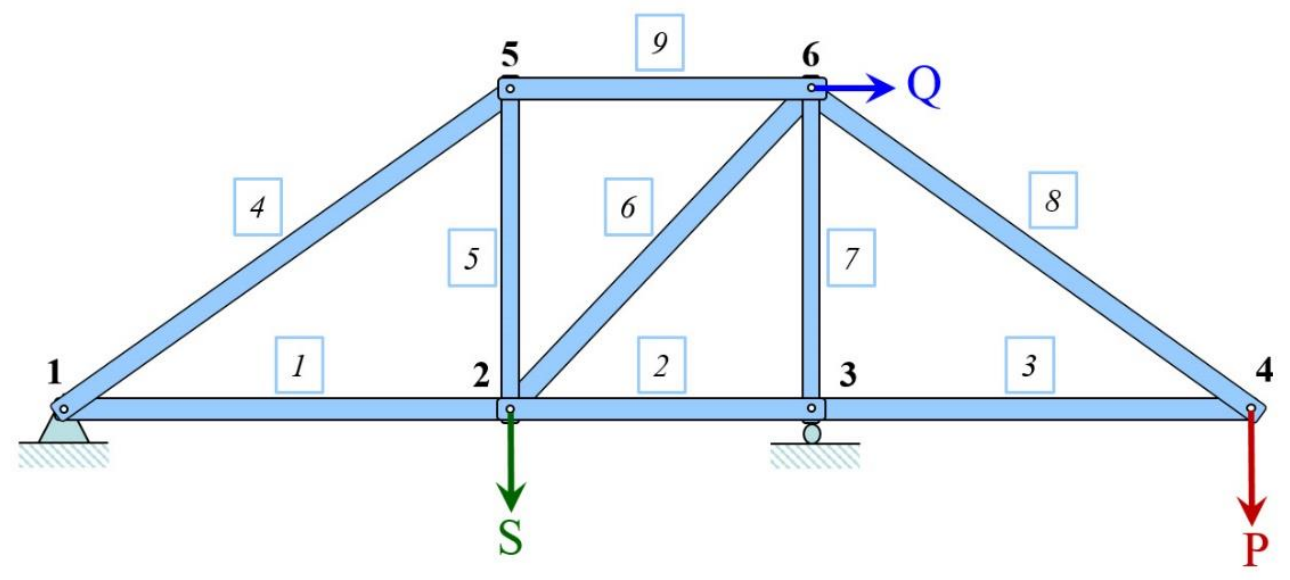

Figure 5. Truss with loads $\mathrm{P}, \mathrm{Q}$ and S.

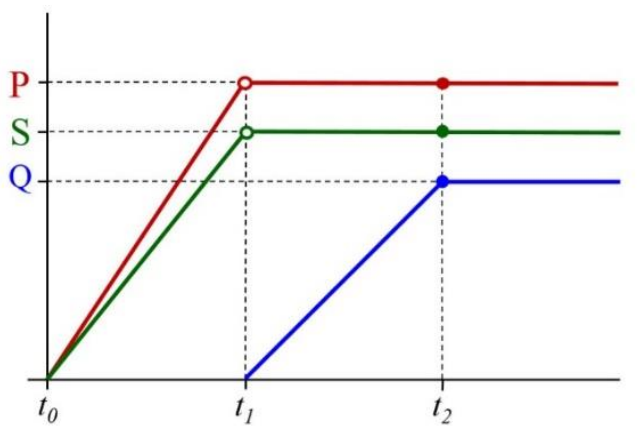

Load Sequence I

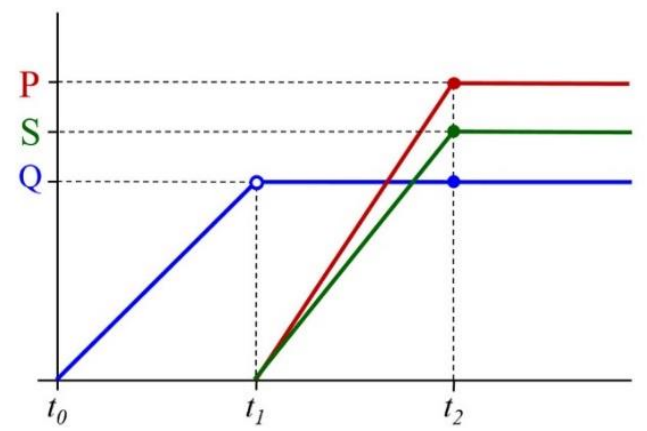

Load Sequence II

Figure 6. Loads P, Q and S applied with Load Sequences I and II.

Similar to the previous situation, we wish to determine the horizontal displacement at joint 6 that is now due entirely to the loads $\mathrm{P}$ and $\mathrm{S}$. By developing the necessary expression to obtain the desired displacement for this loading condition, the influence of the load $\mathrm{S}$ can be easily generalized to develop a displacement equation for a truss with any number of external loads.

Referring to the load sequences in Figure 6, the load $\mathrm{S}$ is applied at the same time as the load $\mathrm{P}$. In Figure 6, notice at $t_{2}$ and beyond the loads $\mathrm{P}, \mathrm{Q}$ and $\mathrm{S}$ are all fully applied for both loading sequences. Because of this condition at $t_{2}$ and beyond, each member will have an axial force and corresponding strain from load sequence I that is equal to the member's axial force and strain from load sequence II. Thus as before, since the strain energy is equal to the total external work 
for each loading sequence ( $U_{I}=W_{I}$ and $\left.U_{I I}=W_{I I}\right)$, we can equate the strain energy from load sequence I with the total external work from load sequence II $\left(U_{I}=W_{I I}\right)$ at $t_{2}$ and beyond. We will use this relationship to find an expression for the horizontal displacement at joint 6 that is due entirely to the loads P and S.

First, considering the strain energy from load sequence I, Equation (5) is updated to include the additional strain energy stored in the members due to the load $\mathrm{S}$ from $t_{0}$ to $t_{2}$.

$$
\begin{gathered}
U_{I}=\frac{1}{2} \sum_{j=1}^{9} F_{j}^{P} \delta_{j}^{P}+\frac{1}{2} \sum_{j=1}^{9} F_{j}^{S} \delta_{j}^{S}+\frac{1}{2} \sum_{j=1}^{9} F_{j}^{Q} \delta_{j}^{Q} \\
+\sum_{j=1}^{9} F_{j}^{P} \delta_{j}^{Q}+\sum_{j=1}^{9} F_{j}^{S} \delta_{j}^{Q}
\end{gathered}
$$

where $F_{j}^{S}$ is the internal force in member $j$ due to the load $\mathrm{S}$ and $\delta_{j}^{S}$ is the change in length of member $j$ due to $F_{j}^{S}$. The last two terms in Equation (12) result from the fact that the loads $\mathrm{P}$ and $\mathrm{S}$ are already fully applied when they are multiplied by the change in member lengths $\delta_{j}^{Q}$ (due to load Q) to account for this portion of the strain energy from $t_{1}$ to $t_{2}$.

Next, considering the external work from load sequence II, Equation (7) is updated to include the additional external work due to the load $\mathrm{S}$ from $t_{0}$ to $t_{2}$.

$$
W_{I I}=\frac{1}{2} Q \Delta_{66}+\frac{1}{2} S \Delta_{22}+\frac{1}{2} P \Delta_{44}+Q \Delta_{64}+Q \Delta_{62}
$$

where $\Delta_{62}$ is the horizontal displacement at joint 6 due to the load $\mathrm{S}$ at joint 2 . The last two terms in Equation (13) result from the fact that the load Q is already fully applied when it is multiplied by the displacements $\Delta_{64}$ (due to load P) and $\Delta_{62}$ (due to load S) to account for this portion of external work from $t_{1}$ to $t_{2}$.

Just as with Equations (4) and (8), conservation of energy provides the following relationship for the application of load $\mathrm{S}$ in load sequence II from $t_{0}$ to $t_{1}$.

$$
\frac{1}{2} \sum_{j=1}^{9} F_{j}^{S} \delta_{j}^{S}=\frac{1}{2} S \Delta_{22}
$$

As previously mentioned, we equate the strain energy from load sequence I with the total external work from load sequence II $\left(U_{I}=W_{I I}\right)$ at $t_{2}$ and beyond to find an expression for the horizontal displacement at joint 6 that is due entirely to the loads P and S. Using Equation (12) for $U_{I}$, and Equation (13) for $W_{I I}$, we obtain the following relationship

$$
\begin{array}{r}
\frac{1}{2} \sum_{j=1}^{9} F_{j}^{P} \delta_{j}^{P}+\frac{1}{2} \sum_{j=1}^{9} F_{j}^{S} \delta_{j}^{S}+\frac{1}{2} \sum_{j=1}^{9} F_{j}^{Q} \delta_{j}^{Q}+\sum_{j=1}^{9} F_{j}^{P} \delta_{j}^{Q}+\sum_{j=1}^{9} F_{j}^{S} \delta_{j}^{Q} \\
=\frac{1}{2} Q \Delta_{66}+\frac{1}{2} S \Delta_{22}+\frac{1}{2} P \Delta_{44}+Q \Delta_{64}+Q \Delta_{62}
\end{array}
$$


Due to the equalities given in Equations (4), (8) and (14), this expression simplifies to the following relationship

$$
\sum_{j=1}^{9} F_{j}^{P} \delta_{j}^{Q}+\sum_{j=1}^{9} F_{j}^{S} \delta_{j}^{Q}=Q \Delta_{64}+Q \Delta_{62}
$$

Using the principle of superposition, Equation (16) can be written in the following form

$$
\sum_{j=1}^{9} F_{j}^{P+S} \delta_{j}^{Q}=Q \Delta_{6}
$$

where $F_{j}^{P+S}=F_{j}^{P}+F_{j}^{S}$ and $\Delta_{6}=\Delta_{64}+\Delta_{62}$.

From Equation (17), we recognize that $F_{j}^{P+S}$ is the sum of the internal forces due to the loads $\mathrm{P}$ and S. Since they are applied simultaneously in Figure 6, there is no need to evaluate $F_{j}^{P}$ and $F_{j}^{S}$ separately; only one analysis of the truss is necessary to obtain $F_{j}^{P+S}$ directly. The change in member lengths $\delta_{j}^{Q}$ (due to the load Q) are determined from a separate analysis using Equation (11). The desired displacement $\Delta_{6}$ is the sum of the contributions to the horizontal displacement due to the load $\mathrm{P}$ at joint 4 and the load $\mathrm{S}$ at joint 2 . There is no need to evaluate $\Delta_{64}$ and $\Delta_{62}$ separately as the desired displacement $\Delta_{6}$ is obtained directly from Equation (17).

Referring to Equation (17) and the values that are necessary to obtain the displacement $\Delta_{6}$, it is noticed that the member forces $F_{j}^{P+S}$ are determined independently from any influence of the load $\mathrm{Q}$, and from Equation (11) it is noticed that the change in member lengths $\delta_{j}^{Q}$ are determined independently from any influence of the loads $\mathrm{P}$ and $\mathrm{S}$. With this being the case we also see from Figure 6 that $F_{j}^{P+S}$ and $\delta_{j}^{Q}$ can be determined from either load sequence I or II at $t_{2}$ and beyond, and the results for both will be identical. All of these conditions allow us to dispense with the formality of considering the actual load sequences in Figure 6 since we can find all that is necessary in Equation (17) from just the two separate analyses considering the loads at only $t_{2}$ and beyond.

\section{Displacement at a Desired Location Due to Any Number of External Loads}

Recognizing we could have any number of external loads and their displacement contributions would all be multiplied by Q, and also that the internal forces are determined from just one analysis with all the external forces applied simultaneously, we can write the following generalized expression for displacement due to any number of external loads.

$$
\sum_{j=1}^{n} F_{j}^{k} \delta_{j}^{Q}=Q \Delta
$$


where $n$ is the number of truss members, $F_{j}^{k}$ is the internal member forces due to all external loads applied simultaneously, $\delta_{j}^{Q}$ is the change in member lengths due to the load $\mathrm{Q}$, and $\Delta$ is the desired displacement at the location and in the direction of the load Q.

The displacement $\Delta$ in Equation (18) is due to all the external loads except Q. Since this is the case, $Q$ can be any magnitude provided simple truss behavior remains valid. Furthermore, the load Q does not actually need to be one of the loads from the actual loading condition of the truss. It can exist solely as a convenience to find the displacement anywhere on the truss where one is desired. Because it does not need to be part of the actual loading condition, Q is often referred to as a virtual force, and the work that it does in Equation (18) is often referred to as virtual work. For the sake of convenience, $\mathrm{Q}=1$ is often used, and the change in member lengths $\delta_{j}^{Q}$ are calculated using Equation (11) with member forces $F_{j}^{Q}$ that are due to this "unit load".

For the case in which a desired displacement is at the location and in the direction of a load that is part of the actual loading condition, Equation (18) can still be used directly. In this case the load Q is applied along with the other external loads to calculate the internal member forces $F_{j}^{k}$. The change in member lengths $\delta_{j}^{Q}$ can be calculated based on the actual load Q (in which case the actual magnitude of load $\mathrm{Q}$ is used in Equation (18)), or $\delta_{j}^{Q}$ can be based on member forces $F_{j}^{Q}$ that are due to a unit load Q (in which case $\mathrm{Q}=1$ is used in Equation (18)).

\section{Example Used for Instruction}

The three member truss given in Figure 7 was used to introduce the real work and strain energy method in my Mechanics of Materials course. The desired displacement is the horizontal displacement at joint 3 that is due entirely to the load $\mathrm{P}=15 \mathrm{kips}$ at joint 2 . A load $\mathrm{Q}=8$ kips is used to find the horizontal displacement $\Delta_{32}^{P}$.

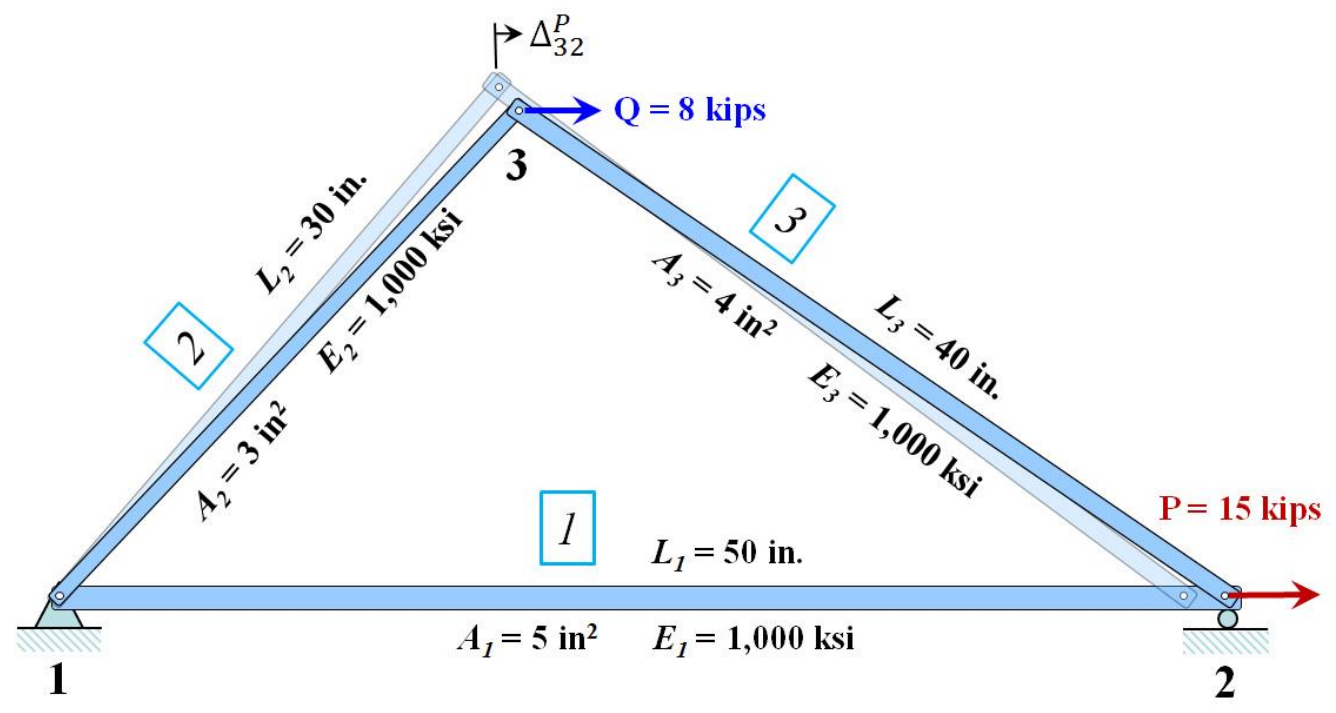

Figure 7. Three member truss with loads $\mathrm{P}$ and $\mathrm{Q}$. 
In Figure 8, notice the loads $\mathrm{P}$ and $\mathrm{Q}$ are fully applied at $t_{2}$ and beyond for both load sequences I and II. As previously discussed at $t_{2}$ and beyond, each member has an axial force and corresponding strain from load sequence I that are equal to the member's axial force and strain from load sequence II, thus $U_{I}=U_{I I}$. Since $W_{I}=U_{I}$ and $W_{I I}=U_{I I}$, then $W_{I I}=U_{I}$ at $t_{2}$ and beyond. It is this last relationship that allows us to write the expression for the displacement $\Delta_{32}^{P}$ that is due entirely to the load P.

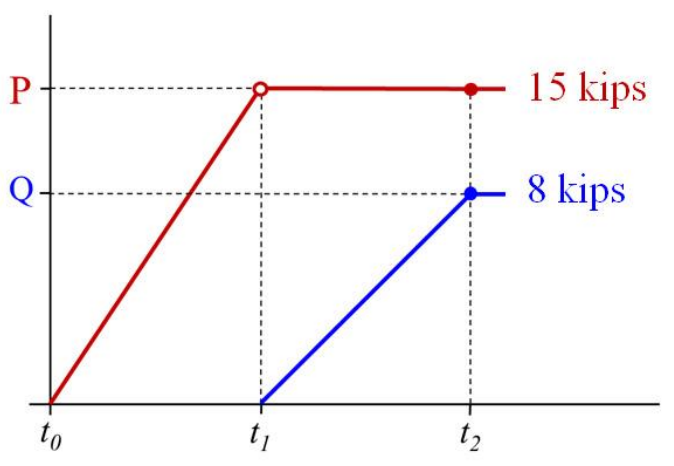

Load Sequence I

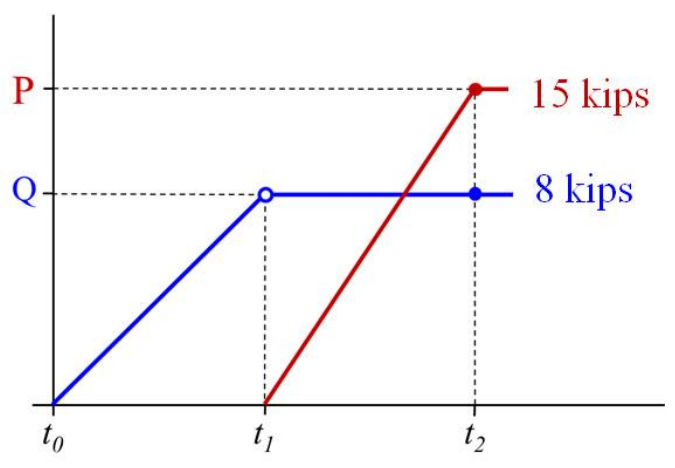

Load Sequence II

Figure 8. Loads P and Q Applied with Load Sequences I and II.

The external work and strain energy equations at $t_{1}$ and $t_{2}$ are given in Table 1 . An analysis of the truss at $t_{1}$ reveals $F_{2}^{P}=F_{3}^{P}=0$, thus the strain energy $U_{I}$ due to $\mathrm{P}$ is zero for these two members. The external work $W_{I I}$ at $t_{2}$ and beyond includes the work that is done by applying the load $\mathrm{P}$ at joint 2 and the additional work due to the load Q that is already fully applied at joint 3 . The strain energy $U_{I}$ at $t_{2}$ and beyond includes the strain energy that is done by applying the load Q and the additional strain energy due to load $\mathrm{P}$ that is already fully applied creating member force $F_{1}^{P}$. As demonstrated in Table 2 , the terms in Table 1 can be rearranged according to the external work and strain energy equalities discussed in the previous sections of the paper. The three equations in Table 2 provide for the determination of $\Delta_{33}^{Q}, \Delta_{22}^{P}$ and $\Delta_{32}^{P}$. The third equation is the only expression that is needed to find the desired displacement $\Delta_{32}^{P}$.

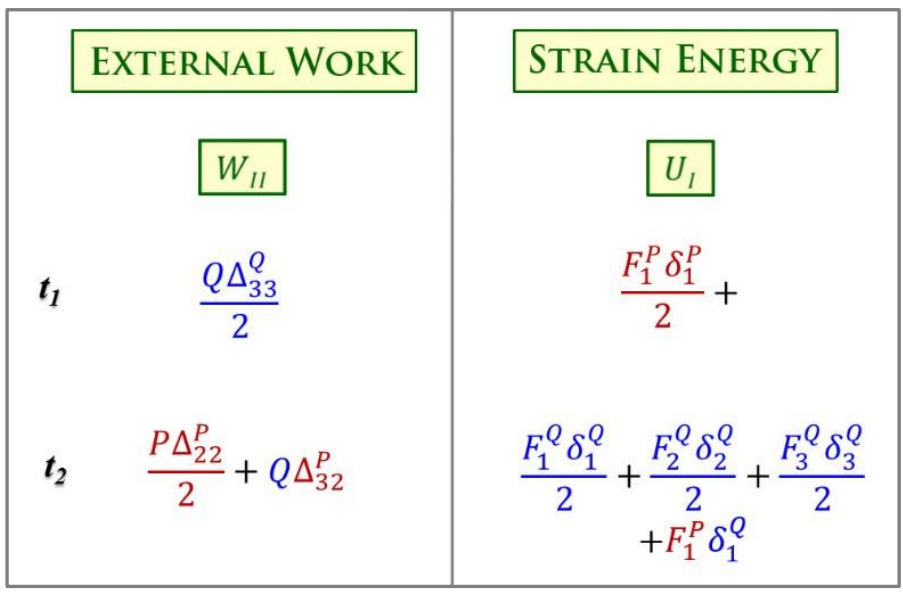

Table $1 . W_{I I}$ and $U_{I}$ for the three member truss in Figure 7. 


\begin{tabular}{rlc|}
$W_{I I}$ & $=$ & $U_{I}$ \\
$\frac{Q \Delta_{33}^{Q}}{2}$ & $=$ & $\frac{F_{1}^{Q} \delta_{1}^{Q}}{2}+\frac{F_{2}^{Q} \delta_{2}^{Q}}{2}+\frac{F_{3}^{Q} \delta_{3}^{Q}}{2}$ \\
$\frac{P \Delta_{22}^{P}}{2}$ & $=$ & $\frac{F_{1}^{P} \delta_{1}^{P}}{2}$ \\
$Q \Delta_{32}^{P}$ & $=$ & $F_{1}^{P} \delta_{1}^{Q}$
\end{tabular}

Table 2. $W_{I I}$ and $U_{I}$ equalities for the three member truss in Figure 7.

For this example problem, we only need to determine the member force $F_{1}^{P}$ and the change in member length $\delta_{1}^{Q}$ in order to determine $\Delta_{32}^{P}$. An analysis of the truss with just the load P gives a member force $F_{1}^{P}=15 \mathrm{kips}$, and a separate analysis with just the load Q gives a member force $F_{1}^{Q}=5.12$ kips. The change in member length $\delta_{1}^{Q}$ is found by using Equation (11).

$$
\delta_{1}^{Q}=\frac{F_{1}^{Q} L_{1}}{A_{1} E_{1}}=\frac{5.12(50)}{5(1,000)}=0.0512 \mathrm{in} .
$$

Finally, using the third equation in Table 2, the horizontal displacement that is due exclusively to the load $\mathrm{P}$ is

$$
\rightarrow \Delta_{32}^{P}=\frac{15(0.0512)}{8}=0.096 \mathrm{in} .
$$

As a follow-up to this example, the vertical displacement at joint 3 that is due exclusively to the load $\mathrm{P}$ is considered, but this time the two load sequences and all three external work and strain energy equations are not used to determine the desired displacement.

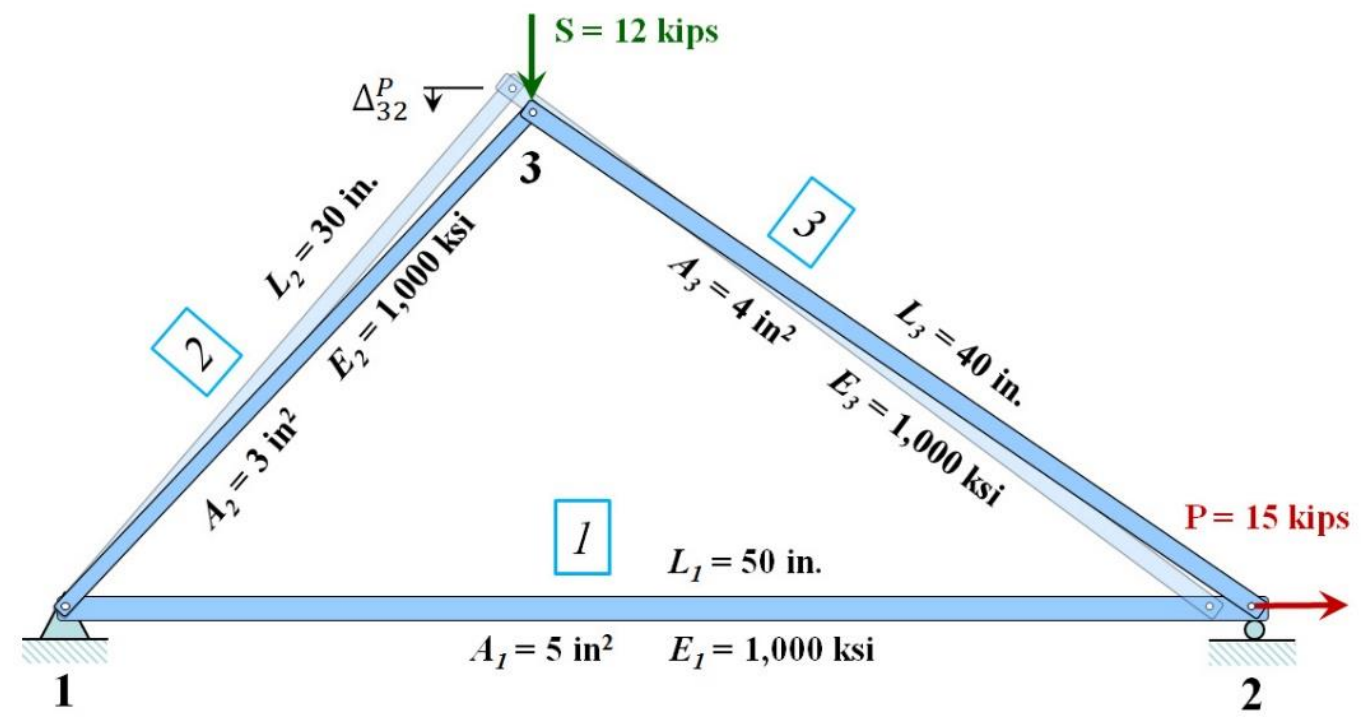

Figure 9. Three member truss with loads $\mathrm{P}$ and $\mathrm{S}$. 
As given in Figure 9, a vertical load $\mathrm{S}=12 \mathrm{kips}$ is used at joint 3 to find the desired displacement. As the previous example demonstrated, the following equation is the only relationship that is needed to determine the vertical displacement $\Delta_{32}^{P}$.

$$
S \Delta_{32}^{P}=F_{1}^{P} \delta_{1}^{S}
$$

An analysis with just the load $S$ gives a member force $F_{1}^{S}=5.76$ kips. Equation (11) is used to determine $\delta_{1}^{S}=0.0576$ in., and the vertical displacement due exclusively to the load $\mathrm{P}$ is

$$
\downarrow \Delta_{32}^{P}=\frac{15(0.0576)}{12}=0.072 \mathrm{in} .
$$

\section{Survey of Students' Understanding of Concepts}

In order to ascertain the students' perceived level of understanding of the real work and strain energy method versus the traditional virtual work method, a lecture was given presenting both methods using the truss and loads in Figures 7 and 9. Throughout the lecture, the students were asked to respond to the 9 survey questions given in Table 3. Each question was displayed on the screen only after that particular portion of the presentation was completed. The students were asked to respond with a $\mathbf{1}$ if they "agreed" with the statement, a $\mathbf{2}$ if they "somewhat agreed", a $\mathbf{3}$ if they "somewhat disagreed", and a $\mathbf{4}$ if they "disagreed". The survey was completely voluntary and anonymous. A total of 55 students in my Mechanics of Materials course completed the survey. All of the students had prior knowledge of work and energy principles from their Physics courses, but none had any prior experience with the virtual work method.

First, the virtual work method was presented using the same terminology as found in Hibbeler ${ }^{1}$. The loads used in the virtual work example were $\mathrm{P}=15$ kips and $\mathrm{Q}=1$ kip. Referring to Table 3, the students were asked to respond to survey questions 1 and 2 during this part of the presentation. Next, the real work and strain energy method was presented using $\mathrm{P}=15$ kips and $\mathrm{Q}=8$ kips. The students were asked questions 3 through 8 during this part of the presentation. Finally, using $\mathrm{P}=15$ kips and $\mathrm{S}=12$ kips, the students were asked survey question 9 .

Comparing the mean results of survey question 2 with question 8, students found the virtual work method to be more difficult to understand as to why the displacement at joint 3 can be found using $\mathrm{Q}$ when compared with the real work and strain energy method. Mean responses to survey questions 3, 4 and 5 indicate that the students generally understood the development of the real work and strain energy method. Mean responses to survey questions 6 and 7 indicate that although the load sequences help to explain why $Q \Delta_{32}^{P}$ contributes to the external work and $F_{1}^{P} \delta_{1}^{Q}$ contributes to the strain energy, they remain slightly more difficult concepts to understand. As these two concepts are key to understanding the virtual work method, extra care is needed when explaining these concepts. The mean response to survey question 9 seems to indicate that once the students have been introduced to the real work and strain energy method, they are able to more easily understand why the virtual work method is able to provide the necessary equation to determine the desired displacement. 
Mean Std.Dev.

1. "I understand how the internal virtual forces $F$ ride along the $\quad \begin{array}{rl}\mathbf{2 . 8 7} & \mathbf{0 . 7 9 5}\end{array}$ change in member lengths $\delta . "$

2. "I understand why the horizontal displacement at $\mathbf{3}$ (that is due exclusively to $P$ ) can be found by applying $Q=1$ with the virtual work method."

3. "I understand how to calculate the displacement of an axial force member using strain energy and external work."

4. "I understand how the two forces P and Q are applied to the truss."

5. "I understand why the external work and strain energy are all equal at $t_{2}$ for both load sequences."

6. "I understand why the external work expression at $t_{2}$ includes the term $Q \Delta_{32}^{P}$."

7. "I understand why the strain energy expression at $t_{2}$ includes the term $F_{1}^{P} \delta_{1}^{Q}$."

8. "I understand why the horizontal displacement at $\mathbf{3}$ (that is due exclusively to $P$ ) can be found by applying $Q=8$ with the real work and strain energy method."

9. "I understand why the vertical displacement at $\mathbf{3}$ (that is due $1.78 \quad 0.875$

$1.53 \quad 0.790$

$1.53 \quad 0.766$

$2.07 \quad 0.858$

1.910 .823 exclusively to $\mathrm{P}$ ) is found by applying $\mathrm{S}$ without needing to see all the strain energy and external work expressions as before."

\section{$1.58 \quad 0.658$}

Table 3. Survey questions with mean and standard deviation results $(n=55)$.

\section{References}

1. R. C. Hibbeler, Mechanics of Materials ( $9^{\text {th }}$ Edition), Pearson Prentice Hall, Upper Saddle River, NJ, 2014.

2. J. M. Gere, B. J. Goodno, Mechanics of Materials ( $8^{\text {th }}$ Edition), Cengage Learning, Stamford, CT, 2013. 
3. F. P. Beer, E. R. Johnston, J. T. DeWolf, D. F. Mazurek, Mechanics of Materials $\left(7^{\text {th }}\right.$ Edition), McGraw-Hill, New York, NY, 2014.

4. W. F. Riley, L. D. Sturges, D. H. Morris, Mechanics of Materials (6 ${ }^{\text {th }}$ Edition), John Wiley \& Sons, Hoboken, NJ, 2007.

5. R. R. Craig, Mechanics of Materials ( ${ }^{\text {rd }}$ Edition), John Wiley \& Sons, Hoboken, NJ, 2011.

6. T. A. Philpot, Mechanics of Materials: An Integrated Learning System ( $3^{\text {rd }}$ Edition), John Wiley \& Sons, Hoboken, NJ, 2013.

7. R. C. Hibbeler, Structural Analysis ( $9^{\text {th }}$ Edition), Pearson Prentice Hall, Upper Saddle River, NJ, 2015.

8. K. M. Leet, C. M. Uang, A. M. Gilbert, Fundamentals of Structural Analysis (4 ${ }^{\text {th }}$ Edition), McGraw-Hill, New York, NY, 2010.

9. A. Kassimali, Structural Analysis ( $5^{\text {th }}$ Edition), Cengage Learning, Stamford, CT, 2015. 


\section{Appendix A}

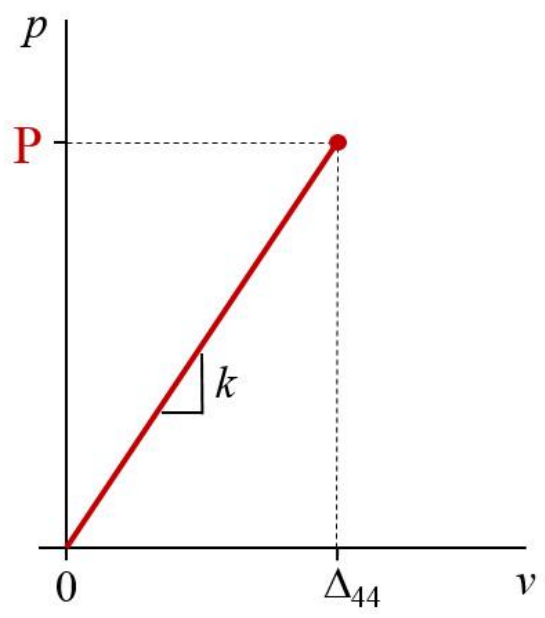

Figure A.1. Load vs. displacement diagram for Load Sequence I in Figure 2.

For the truss in Figure 1 with the load applied at joint 4 according to the loading sequence in Figure 2, the external work that is done from $t_{0}$ to $t_{1}$ is

$$
W=\int_{0}^{\Delta_{44}} p d v
$$

With the linear-elastic material properties in Figure A.1, $p=k \cdot v$ and $k=P / \Delta_{44}$. Equation (A.1) can be written as

$$
W=\int_{0}^{\Delta_{44}} \frac{P}{\Delta_{44}} v d v
$$

where $\Delta_{44}$ is the vertical displacement at joint 4 due to the load $\mathrm{P}$ at joint 4 . Evaluating the integral in Equation (A.2) gives the final expression for the external work as

$$
W=\frac{1}{2} P \Delta_{44}
$$

The strain energy stored in each member of the truss $j$ from $t_{0}$ to $t_{1}$ is determined considering a volume element where the force on the top and bottom area $d A$ is $d F_{j}^{P}=\sigma_{j}^{P} d A$, and along the element length $d z$ a change in length occurs that is $d \delta_{j}^{P}=\epsilon_{j}^{P} d z$. The stress $\sigma_{j}^{P}$ and the strain $\epsilon_{j}^{P}$ in each member $j$ are due to the application of load from zero to $\mathrm{P}$ as given in Figure 2 , thus the internal work done by $d F_{j}^{P}$ in each member is $d U_{j}^{P}=1 / 2 d F_{j}^{P} d \delta_{j}^{P}=1 / 2 \sigma_{j}^{P} d A \epsilon_{j}^{P} d z$. Thus considering all members of the truss, the strain energy is

$$
U=\sum_{j=1}^{9} \int_{V} \frac{\sigma_{j}^{P} \epsilon_{j}^{P}}{2} d V
$$


Since $\sigma_{j}^{P}=F_{j}^{P} / A_{j}$ and $\epsilon_{j}^{P}=\sigma_{j}^{P} / E_{j}$, Equation (A.4) can be written as

$$
U=\sum_{j=1}^{9} \int_{0}^{L_{j}} \frac{\left(F_{j}^{P}\right)^{2}}{2 A_{j} E_{j}} d z=\sum_{j=1}^{9} \frac{\left(F_{j}^{P}\right)^{2} L_{j}}{2 A_{j} E_{j}}
$$

where $F_{j}^{P}$ is the internal force in member $j$ due to the load P. The change in length of member $j$ due to $F_{j}^{P}$ is determined using $d \delta_{j}^{P}=\epsilon_{j}^{P} d z$ and the expressions for $\sigma_{j}^{P}$ the $\epsilon_{j}^{P}$ given above.

$$
\delta_{j}^{P}=\int_{0}^{L_{j}} \frac{F_{j}^{P}}{A_{j} E_{j}} d z=\frac{F_{j}^{P} L_{j}}{A_{j} E_{j}}
$$

Substituting Equation (A.6) into Equation (A.5), the strain energy in the truss given in Figure 1 due to the load sequence in Figure 2 is

$$
U=\frac{1}{2} \sum_{j=1}^{9} F_{j}^{P} \delta_{j}^{P}
$$




\section{Appendix B}
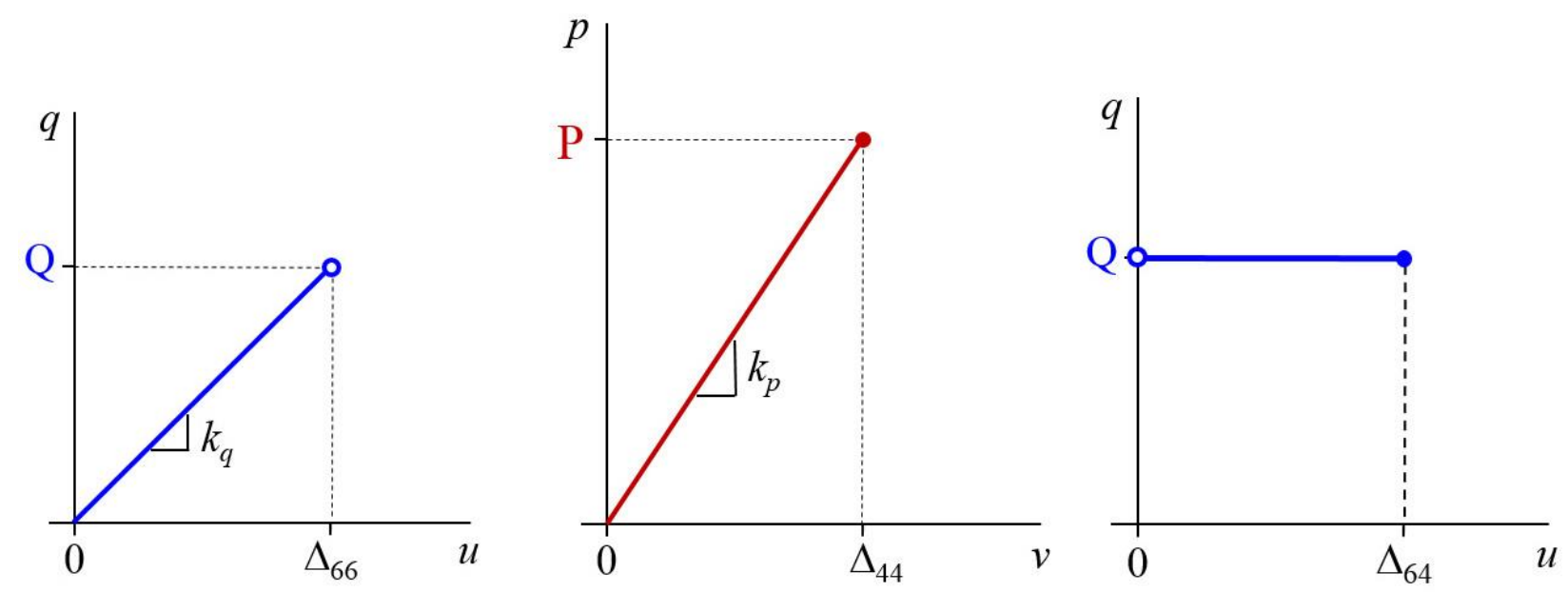

Figure B.1. Load vs. displacement diagrams for Load Sequence II in Figure 4.

For the truss in Figure 3 with the loads applied according to load sequence II in Figure 4, the external work that is done from $t_{0}$ to $t_{1}$ is

$$
W_{I I}=\int_{0}^{\Delta_{66}} q d u
$$

where $\Delta_{66}$ is the horizontal displacement at joint 6 due to the load Q at joint 6 . The additional external work from $t_{1}$ to $t_{2}$ is

$$
W_{I I}=\int_{0}^{\Delta_{44}} p d v+\int_{0}^{\Delta_{64}} Q d u
$$

where $\Delta_{44}$ is the vertical displacement at joint 4 due to the load P at joint 4 , and $\Delta_{64}$ is the horizontal displacement at joint 6 due to the load $\mathrm{P}$ at joint 4 . Referring to the second integral in Equation (B.2), the load Q is already fully applied from $t_{1}$ to $t_{2}$ and is therefore constant when the horizontal displacement at joint 6 occurs due to the application of the load P. Thus, the total external work for load sequence II from $t_{0}$ to $t_{2}$ is

$$
W_{I I}=\int_{0}^{\Delta_{66}} q d u+\int_{0}^{\Delta_{44}} p d v+\int_{0}^{\Delta_{64}} Q d u
$$

With the linear-elastic material properties in Figure B.1, $q=k_{q} \cdot u, k_{q}=Q / \Delta_{66}, p=k_{p} \cdot v$ and $k_{p}=P / \Delta_{44}$, Equation (B.3) can be written as

$$
W_{I I}=\int_{0}^{\Delta_{66}} \frac{Q}{\Delta_{66}} u d u+\int_{0}^{\Delta_{44}} \frac{P}{\Delta_{44}} v d v+Q \Delta_{64}
$$


Evaluating the integrals in Equation (B.4), the final expression for the external work from $t_{0}$ to $t_{2}$ is

$$
W_{I I}=\frac{1}{2} Q \Delta_{66}+\frac{1}{2} P \Delta_{44}+Q \Delta_{64}
$$

For the truss in Figure 3 with the loads applied according to load sequence I in Figure 4, the strain energy stored in each member of the truss $j$ from $t_{0}$ to $t_{1}$ is the same as that given in Appendix A. Thus from Equation (A.7), the strain energy in the truss from $t_{0}$ to $t_{1}$ is

$$
U_{I}=\frac{1}{2} \sum_{j=1}^{9} F_{j}^{P} \delta_{j}^{P}
$$

The additional strain energy from $t_{1}$ to $t_{2}$ is determined considering a volume element where the force on the top and bottom area $d A$ is $d F_{j}^{Q}=\sigma_{j}^{Q} d A$, and along the element length $d z$ a change in length occurs that is $d \delta_{j}^{Q}=\epsilon_{j}^{Q} d z$. The stress $\sigma_{j}^{Q}$ and the strain $\epsilon_{j}^{Q}$ in each member $j$ are due to the application of load from zero to $\mathrm{Q}$ as given in load sequence I in Figure 4. Thus the internal work done by $d F_{j}^{Q}$ in each member is $d U_{j}^{Q}=1 / 2 d F_{j}^{Q} d \delta_{j}^{Q}=1 / 2 \sigma_{j}^{Q} d A \epsilon_{j}^{Q} d z$. Also, from $t_{1}$ to $t_{2}$ the load $\mathrm{P}$ is already fully applied when the change in element length $d \delta_{j}^{Q}$ occurs due to the application of Q. Thus the internal work done by $d F_{j}^{P}$ in each member is $d U_{j}^{P}=d F_{j}^{P} d \delta_{j}^{Q}=$ $\sigma_{j}^{P} d A \epsilon_{j}^{Q} d z$.

The strain energy in the truss from $t_{1}$ to $t_{2}$ is

$$
U_{I}=\sum_{j=1}^{9} \int_{V} \frac{\sigma_{j}^{Q} \epsilon_{j}^{Q}}{2} d V+\sum_{j=1}^{9} \int_{V} \sigma_{j}^{P} \epsilon_{j}^{Q} d V
$$

Since $\sigma_{j}^{Q}=F_{j}^{Q} / A_{j}$ and $\epsilon_{j}^{Q}=\sigma_{j}^{Q} / E_{j}$, the first term of Equation (B.7) can be written as

$$
\sum_{j=1}^{9} \int_{0}^{L_{j}} \frac{\left(F_{j}^{Q}\right)^{2}}{2 A_{j} E_{j}} d z=\sum_{j=1}^{9} \frac{\left(F_{j}^{Q}\right)^{2} L_{j}}{2 A_{j} E_{j}}
$$

where $F_{j}^{Q}$ is the internal force in member $j$ due to the load $\mathrm{Q}$. Since $\sigma_{j}^{P}=F_{j}^{P} / A_{j}$, the second term of Equation (B.7) can be written as

$$
\sum_{j=1}^{9} \int_{0}^{L_{j}} \frac{F_{j}^{P} F_{j}^{Q}}{A_{j} E_{j}} d z=\sum_{j=1}^{9} \frac{F_{j}^{P} F_{j}^{Q} L_{j}}{A_{j} E_{j}}
$$

where $F_{j}^{Q}$ is the internal force in member $j$ due to the load Q.

The change in length of member $j$ due to $F_{j}^{Q}$ is determined using $d \delta_{j}^{Q}=\epsilon_{j}^{Q} d z$ and the expressions for $\sigma_{j}^{Q}$ the $\epsilon_{j}^{Q}$ given above. 


$$
\delta_{j}^{Q}=\int_{0}^{L_{j}} \frac{F_{j}^{q}}{A_{j} E_{j}} d z=\frac{F_{j}^{Q} L_{j}}{A_{j} E_{j}}
$$

Substituting Equations (B.8), (B.9) and (B.10) into Equation (B.7), the strain energy in the truss from $t_{1}$ to $t_{2}$ is

$$
U_{I}=\frac{1}{2} \sum_{j=1}^{9} F_{j}^{Q} \delta_{j}^{Q}+\sum_{j=1}^{9} F_{j}^{P} \delta_{j}^{Q}
$$

Combining the results from Equations (B.6) and (B.11), the final expression for the strain energy in the truss due to load sequence I in Figure 4 from $t_{0}$ to $t_{2}$ is

$$
U_{I}=\frac{1}{2} \sum_{j=1}^{9} F_{j}^{P} \delta_{j}^{P}+\frac{1}{2} \sum_{j=1}^{9} F_{j}^{Q} \delta_{j}^{Q}+\sum_{j=1}^{9} F_{j}^{P} \delta_{j}^{Q}
$$

